\title{
ENTREPRENEURIAT SOCIAL ET PARTICIPATION CITOYENNE
}

\author{
François Brouard \\ Carleton University \\ Sophie Larivet \\ École Supérieure du Commerce Extérieur, France \\ Ouafa Sakka \\ Carleton University
}

\section{RÉSUMÉ}

L'entrepreneuriat social est un concept émergeant, notamment dans les sciences de l'administration. Pourtant, en dehors d'une pratique directoriale spécifique, il est aussi une forme de participation citoyenne trop méconnue. L'objectif de cet article, basé sur une revue de la littérature et une approche théorique, est de présenter le concept d'entrepreneuriat social afin de mieux saisir son positionnement par rapport à la participation citoyenne. L'entrepreneuriat social constitue une forme particulière de participation à l'espace public par l'action, les entreprises sociales agissant au quotidien pour transformer le paysage social. En particulier, cet article souligne le contexte de développement de l'entrepreneuriat social, définit le concept et les notions connexes d'entreprise sociale et d'entrepreneur social, et, enfin, présente une réflexion sur la contribution de l'entrepreneuriat social à la participation citoyenne. L'article montre que l'entrepreneuriat social est une façon pour les citoyens d'agir directement et avec maîtrise sur la société.

\begin{abstract}
Social entrepreneurship is an emerging concept, notably in administrative sciences. However, not only is it a specific managerial practice but it is also a type of citizen participation that is not well-known. The objective of this article, based on a literature review and a theoretical approach, is to present the concept of social entrepreneurship in order to better understand its relation to citizen participation. Social entrepreneurship represents a specific type of citizen participation involving actions. Social enterprises act daily to transform the social landscape. More specifically, this article presents the context of development of social entrepreneurship, proposes a definition of the concept and of other connected notions like "social enterprise" and "social entrepreneur", and, finally, analyzes the contribution of social entrepreneurship to citizen participation. It shows that social entrepreneurship is a way for citizens to act directly and with some power on society.
\end{abstract}

\section{MOTS CLÉS / KEYWORDS}

Entrepreneuriat social; Entrepreneur social; Entreprise sociale; Participation citoyenne / Social entrepreneurship; Social entrepreneur; Social enterprise; Citizen participation 


\section{INTRODUCTION}

La notion de participation des citoyens à l'espace public couvre, d'une part, la participation citoyenne aux expériences organisées par l'État ou par le biais de regroupements de la société civile, et, d'autre part, les initiatives mises en œuvre par les citoyens eux-mêmes. Au niveau de la participation initiée par les citoyens, une forme particulière d'entrepreneuriat, qu'on appellera ci-après " entrepreneuriat social ", présente une avenue de recherche intéressante puisqu'elle vise un objectif autre que ceux de l'entrepreneuriat traditionnel : la création de valeur « sociale ». Ainsi, l'entrepreneuriat social constitue une forme particulière de participation par l'action qui se situe au niveau des entreprises sociales et des entrepreneurs sociaux agissant au quotidien pour transformer le paysage de la société (Nicholls, 2006).

L'entrepreneuriat joue un rôle de premier plan dans le développement économique (Davis, 2002; Haugh, 2007), essentiellement par la création de nouveaux emplois. Il est ainsi possible et souhaitable de générer de nouvelles formes de développement économique local afin de favoriser les objectifs de développement locaux (Boucher, Favreau, Guindon et Hurtubise, 2000; Joyal, 1999; Thompson, Alvy et Lees, 2000). Dans ce contexte, l'entrepreneuriat social joue un rôle particulièrement important par la recherche et la mise en œuvre de solutions innovantes répondant à des problèmes sociaux, tout en mobilisant les citoyens dans ce processus. Toutefois, ce concept est encore mal connu et cela est d'autant plus vrai au Canada et dans le monde francophone (Audet et Julien, 2006; Johnson, 2000). II convient de noter que, même s'il n'est pas nouveau, l'entrepreneuriat social est un concept émergeant qui gagne en popularité autour de la planète (Barendsen et Gardner, 2004; Christie et Honig, 2006; Schlee, Curren et Harich, 2009). L'attribution du Prix Nobel de la Paix 2006 à Mohammad Yunus et à la Graamen Bank est sans contredit un coup de pouce majeur à cette popularité (Renaud, 2007; Yunus, 2006). Mohammad Yunus a créé la Graamen Bank afin de permettre aux femmes d'un village du Bangladesh d'accéder au crédit et de combattre l'injustice et la pauvreté. La Graamen Bank est une institution de microcrédit qui a fait boule de neige dans le monde et qui aide maintenant des centaines de millions de femmes (Anderson, Dana et Dana, 2006; Johnson, 2000).

La présente contribution est une étude conceptuelle et exploratoire visant à mettre en évidence l'apport du concept d'entrepreneuriat social à la notion de participation citoyenne. La suite du texte se divise en trois grandes parties. La première partie souligne le contexte de développement du concept d'entrepreneuriat social. La deuxième partie propose une définition du concept d'entrepreneuriat social et tente de le distinguer d'autres concepts connexes. La troisième partie présente une réflexion sur la contribution de l'entrepreneuriat social à la participation citoyenne. Une conclusion termine le tout.

\section{CONTEXTE DE DÉVELOPPEMENT DE L'ENTREPRENEURIAT SOCIAL}

Pour mieux comprendre le concept d'entrepreneuriat social, il est utile de situer le contexte de développement de ce concept qui, de fait, justifie son importance grandissante (Dees, 1998; Johnson, 2000; National Center for Social Entrepreneurs, 2001). Traditionnellement, les missions associées à l'entrepreneuriat social, telles que fournir des services de support aux femmes, ou éduquer les personnes démunies, sont assumées par les organismes sans but lucratif (secteur bénévole et communautaire) ou par les États eux-mêmes (secteur public). II y 
a donc lieu d'analyser les principales raisons pour lesquelles des entreprises se tournent aujourd'hui vers l'entrepreneuriat social. II faut noter qu'au Canada, le développement de ce type particulier d'entrepreneuriat est encore à un stade embryonnaire avec des variations selon les provinces (Guay et Riverin, 2006; Johnson, 2000; 2003). Les éléments explicatifs évoqués ci-dessous peuvent avoir une importance plus ou moins grande selon les pays ou régions.

Une première raison pour le développement de l'entrepreneuriat social réside dans le besoin de financement des missions sociales. Les gouvernements peuvent décider de diminuer le financement de certains services afin de mieux équilibrer leur budget, ou de changer la répartition de ce financement. Les organismes à but non lucratif à vocation sociale, contrairement aux idées reçues, ne sont que très partiellement financés par des dons privés (fondations, particuliers). Ils dépendent majoritairement des financements publics et, dans une moindre mesure, de leurs autres sources de revenus (par exemple, adhésions, mais aussi revenus d'activités commerciales) (Salamon, Anheier, List, Toepler, Sokolowski et Associates, 1999). Les organisations à vocation sociale ont donc besoin de se prémunir contre les fluctuations éventuelles de leurs financements externes qui peuvent mettre en danger la livraison des services sociaux. Elles doivent développer de nouvelles formes de financement, telles que le financement par des activités commerciales qui est potentiellement plus fiable et stable que les dons et subventions. Cela est d'autant plus vrai dans une période de difficultés économiques qui peut avoir des répercussions importantes pour les organisations sociales (Arts Quarter, 2009).

Une deuxième raison est l'existence de besoins sociaux grandissants. En effet, avec un certain désengagement de l'État dans de nombreux secteurs, les organisations à vocation sociale doivent répondre à des besoins de plus en plus nombreux et diversifiés. Par exemple, de nouveaux besoins ont vu le jour au fil des dernières années, face à des phénomènes tels le sida, les enfants avec des problèmes d'accoutumance à la drogue, et le vieillissement de la population. Ainsi, les changements démographiques ont un effet sur la société en général (Foot et Stoffman, 1996) et posent des défis particuliers au niveau des services et du financement dans une perspective à long terme. Ces changements ajoutent également à la croissance de certains besoins touchant particulièrement la génération des « bebe-boumers ».

Une troisième raison est l'apparition d'organisations plus nombreuses pour répondre aux besoins grandissants. Cette prolifération d'organisations amène une compétitivité accrue pour ce qui est du financement et des services et requiert des solutions innovatrices pour que l'organisation puisse survivre. De plus, des entreprises à but lucratif entrent en compétition avec l'État ou les organisations à but non lucratif pour la livraison de certains services. C'est le cas depuis peu en France, pour l'aide aux chômeurs dans leur recherche d'emploi.

Une quatrième raison est l'appel à la responsabilisation dans l'utilisation des fonds obtenus. Les scandales financiers n'ont pas touché, ces dernières années, que les sphères privée (Enron, Norbourg) et publique (scandale des commandites et Commission Gomery au Canada, notes de frais des élus britanniques). Les organisations à vocation sociale, elles aussi, ont fait la une des journaux pour leur mauvaise gestion ou des affaires délictueuses (scandale de l'ARC en France, de I'UNICEF au Kenya). Une exigence plus grande des bailleurs de fonds et du public face à l'utilisation des fonds publics ou privés s'est développée (Cutt et Murray, 2000) et entraîne une pression pour une meilleure gouvernance. L'efficacité et la proactivité désormais requises des organisations à vocation sociale peuvent les conduire à adopter par isomorphisme des modes de gouvernance proches de ceux des entreprises privées (Queinec, 2007). 
Une cinquième raison est l'acceptation de plus en plus grande du marché comme mécanisme capable de combler les besoins sociaux en faisant appel au pouvoir de la compétition tout en favorisant l'innovation et l'efficacité organisationnelle (Dees, 1998). Cela peut aussi se traduire par une modification importante des infrastructures (Yunus, 2006). Certaines critiques apparaissent toutefois contre le " philanthrocapitalisme »(Edwards, 2008) : le recours aux logiques de marché et l'entrepreneuriat social, notamment à grande échelle, ne sont pas une panacée (par exemple, il existe un risque de vision à court terme) et ne résolvent pas tous les problèmes de la société (notamment le manque de solidarité bénévole).

Ces diverses raisons expliquent l'emballement pour le phénomène de l'entrepreneuriat social depuis quelques années, emballement qui se traduit par les initiatives de nombreux individus et par leur corollaire : la multiplication des travaux académiques tentant de circonscrire et de comprendre le phénomène de l'entrepreneuriat social. La section qui suit tente de définir l'entrepreneuriat social et de le distinguer de certaines notions qui y sont reliées.

\section{ENTREPRENEURIAT SOCIAL : DÉFINITION ET CONCEPTS CONNEXES}

Comme il faut s'y attendre avec un concept en émergence, plusieurs définitions de l'entrepreneuriat social existent dans la littérature (Brouard et Larivet, 2009; Bacq et Janssen, 2008a, 2008b; Zhara, Gadajlovic, Neubaum et Shulman, 2006), et aucune n'est unanimement acceptée. Toutefois, il y a un consensus sur la multidimensionalité de ce concept et sur la présence d'une double filiation, soit l'entrepreneuriat et le social (Mair et Marti, 2006; Nicholls, 2006; Peredo et McLean, 2006; Sullivan Mort, Weerawardena et Carnegie, 2003).

La première facette du concept, "l'entrepreneuriat ", correspond au démarrage d'activité et à la création de valeur pour les entrepreneurs et la société (Peredo et McLean, 2006; Sullivan Mort et al., 2003). L'entrepreneur utilise l'innovation pour saisir des occasions d'affaires et mobilise les ressources à sa disposition pour atteindre des objectifs précis.

La deuxième facette correspond à la mission "sociale " (Brickerhoff, 2000; Tan, Williams et Tan, 2005; Ulhoi, 2005) qui représente l'élément central distinguant l'entrepreneuriat social de l'entrepreneuriat traditionnel. Sans cette mission, il n'y a vraisemblablement pas d'entrepreneuriat social. La mission sociale peut toutefois être combinée à une mission économique. L'entreprise sociale joue un rôle d'agent de changement social d'une manière consciente et non seulement involontaire ou collatérale (Sullivan Mort et al., 2003).

En nous basant sur des travaux antérieurs (Brouard et Larivet, 2009, p.11), nous proposons la définition suivante :

L'entrepreneuriat social est un concept qui représente l'ensemble des activités et des processus pour créer et soutenir la valeur sociale en utilisant des approches entrepreneuriales et innovantes et en tenant compte des contraintes de l'environnement externe.

La Figure 1 présente un modèle conceptuel qui fournit une explication plus large de la définition de l'entrepreneuriat social. Tout d'abord, une entreprise avec entrepreneuriat social peut avoir une mission à la fois économique et sociale. Cependant, il est essentiel qu'il y ait une prédominance au niveau de la mission sociale parce qu'il s'agit de l'élément central qui distingue ce type d'entreprise (Tan et al., 2005; Ulhoi, 2005). Ensuite, la présence de besoins sociaux signifie pour un entrepreneur social l'existence d'occasions qu'il est possible de saisir. 
La dimension entrepreneuriale amène un souci d'innovation et une attitude proactive quant à la façon de combler ces besoins tout en créant de la valeur ajoutée (Sullivan Mort et al., 2003). L'ensemble de l'entreprise sociale vise ainsi une création de valeur qui contribuerait à la satisfaction des besoins sociaux et à l'initiation d'une certaine transformation sociale.

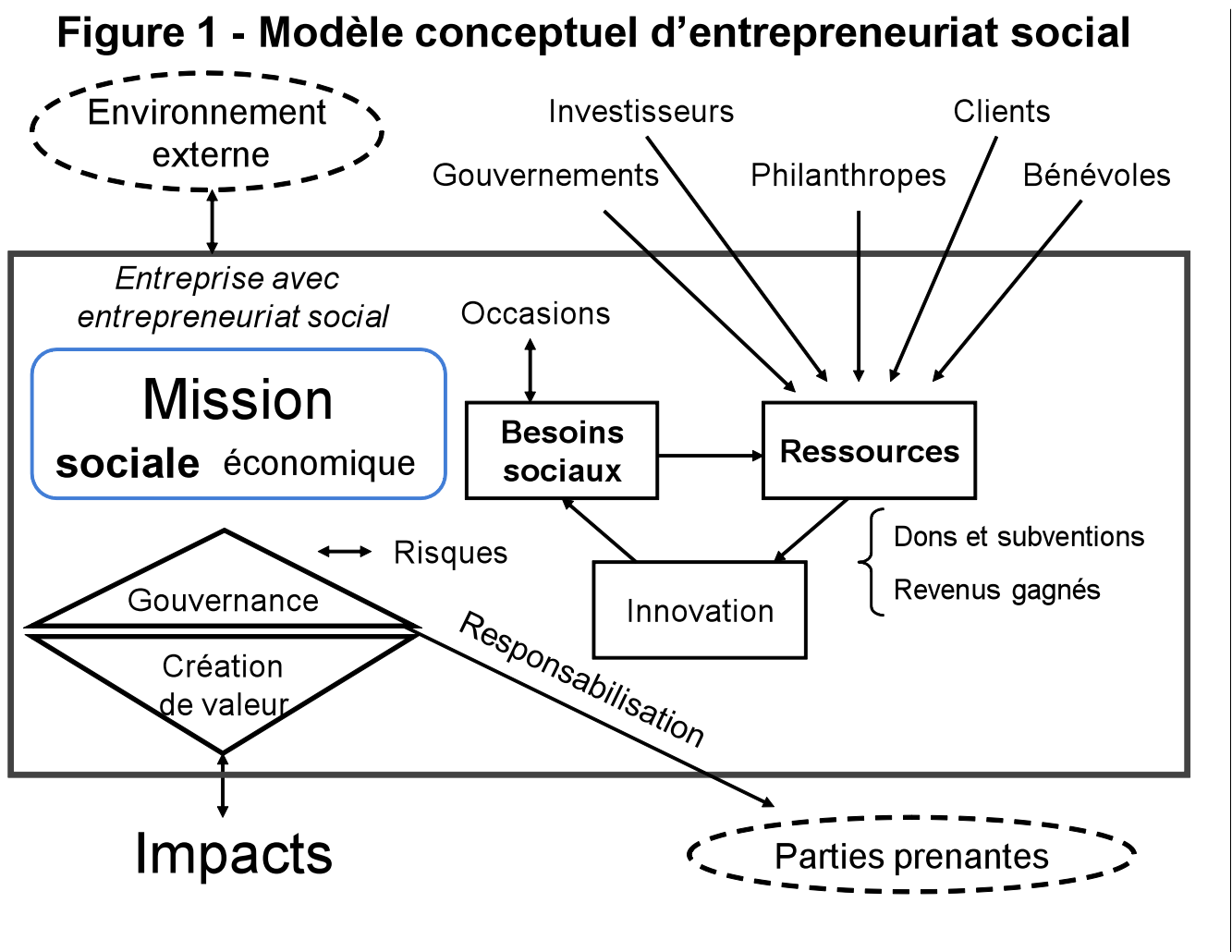

Par ailleurs, les entreprises sociales sont dépendantes de l'environnement externe puisqu'elles obtiennent généralement les ressources financières et humaines des gouvernements, des bailleurs de fonds, des philanthropes et des bénévoles, sous forme de dons et de subventions. De même, les investisseurs et les clients sont des groupes qui doivent être pris en compte par l'entreprise sociale en raison de leur impact sur les ressources obtenues par l'entreprise. En plus de ces sources de financement, l'entreprise est assujettie aux forces qui s'exercent sur elle par le macro-environnement (fiscalité relative aux dons et aux entreprises sociales, innovations technologiques, démographie...) ou par les parties prenantes (clients, employés, gouvernements, médias...). Ainsi, les entreprises sociales doivent, comme les entreprises classiques, surveiller les forces de l'environnement externe et s'y adapter. La gouvernance et la création de valeur sont également des dimensions importantes de l'entrepreneuriat social. En effet, l'entreprise doit pouvoir expliquer et justifier ses actions, tant au niveau économique que social, parce qu'elle aura à en rendre compte à ses financiers et aux différentes parties prenantes.

En examinant le concept d'entrepreneuriat social, il est de mise de distinguer également les concepts d'économie sociale, d'entreprise sociale et d'entrepreneur social. L'entrepreneur social occupe une place privilégiée dans l'entreprise sociale qui elle-même se situe dans l'ensemble de l'économie sociale. II y a toutefois lieu de distinguer ces trois concepts, car ils ne sont pas identiques, même s'ils sont interreliés et parfois utilisés à tort comme des 
synonymes. Ainsi, il est possible de distinguer les différents types d'entreprise sociale selon les secteurs et les types d'organisation.

Selon Painter (2006), trois grands secteurs peuvent être mentionnés, soit le secteur public et le secteur privé aux deux extrêmes et l'économie sociale, incluant les ONG (organisations non gouvernementales). Le secteur public fait référence aux gouvernements fédéraux, provinciaux et territoriaux et aux administrations locales (entités municipales). De par leur nature même, plusieurs entités du secteur public visent une mission sociale et certaines pourraient donc être considérées comme des entreprises sociales. Le secteur privé comprend les entités visant la maximisation du bénéfice.

L'économie sociale se définit comme "l'évolution de diverses combinaisons d'organisations non gouvernementales (ONG) produisant et offrant des biens et services dans les collectivités au Canada et ailleurs dans le monde depuis plus d'un siècle " (Painter, 2006, p.30). L'économie sociale peut être vue comme une action collective axée autour de trois dimensions-sociale, économique et politique-qui donne la formule " s'associer pour entreprendre autrement "(Favreau, 2005). Reprenant la synthèse de Favreau (2005), il est possible de cerner ces trois dimensions : 1) "S'associer " permet de répondre à des besoins socioéconomiques, socioculturels et sociopolitiques de se regrouper dans des organisations démocratiques (c'est-à-dire où les décisions de gestion respectent le principe général « une personne, une voix ", même si ce principe souffre parfois d'exceptions); 2) "Entreprendre " permet d'affronter le marché dans la production de biens et services; 3) " Autrement » permet la pluralité d'engagements citoyens avec des mobiles sociopolitiques divers. L'entrepreneur social ne s'attarde pas nécessairement sur la dimension politique et l'aspect démocratique, mais porte une attention prépondérante au volet " entreprendre " pour répondre à des besoins sociaux.

II y a aussi lieu de distinguer l'entreprise sociale et l'entrepreneur social. L'entrepreneur social est la personne ou le groupe de personnes qui agit à titre de catalyseur de l'entreprise sociale et met à profit ses habiletés entrepreneuriales pour faire avancer l'entreprise sociale. Les entrepreneurs sociaux ne sont pas des gens " raisonnables " selon les critères habituels de l'économie (Elkington et Hartigan, 2008); ils prennent des risques que d'autres ne prendraient pas parce qu'ils croient fermement en la capacité de chacun à contribuer au développement et préfèrent inventer des solutions innovantes plutôt que de se résigner aux lourdeurs bureaucratiques qui freinent les changements sociaux. Ils sont impatients, visionnaires, ambitieux et osent voir des sources de profit là où le marché est réputé inefficace. Ils osent penser la combinaison de l'économique et du social, et ils osent la mettre en œuvre. Ils tentent de changer le monde qui les entoure pour l'adapter à leurs convictions optimistes (Bornstein, 2007; Elkington et Hartigan, 2008).

Le Tableau A présente brièvement une comparaison des entrepreneurs sociaux et des entrepreneurs économiques (ou traditionnels). Les dimensions de cette comparaison sont: les forces, l'accent, la perspective temporelle, l'étendue des produits et services, les bénéfices, les risques et le besoin d'autonomie. 


\section{Tableau A - Comparaison des types d'entrepreneurs}

\begin{tabular}{|l|l|l|}
\cline { 2 - 3 } \multicolumn{1}{l|}{} & Entrepreneurs sociaux & Entrepreneurs économiques \\
\hline Forces & expérience collective & habileté et énergie personnelle \\
\hline Accent & développement de capacités & gain financier \\
\hline $\begin{array}{l}\text { Perspective } \\
\text { Eemporelle } \\
\text { produits et services }\end{array}$ & long terme & court terme \\
\hline \multirow{2}{*}{ Bénéfices } & le profit est un moyen & aucune limite \\
\cline { 2 - 3 } & réinvestissement & le profit est une fin \\
\hline Risques & $\begin{array}{l}\text { actifs de l'organisation, image } \\
\text { et confiance }\end{array}$ & $\begin{array}{l}\text { actifs personnels et des } \\
\text { investisseurs }\end{array}$ \\
\hline \multirow{2}{*}{ Autonomie } & $\begin{array}{l}\text { rendre l'organisation non } \\
\text { dépendante de donateurs }\end{array}$ & $\begin{array}{l}\text { prendre sa destinée en main } \\
\text { plutôt que de dépendre d'un } \\
\text { employeur }\end{array}$ \\
\hline
\end{tabular}

Source : National Center for Social Entrepreneurs (2001, p. 5)

Certains entrepreneurs sociaux sont extrêmement célèbres. Par exemple, dans le domaine de l'éducation, Maria Montessori a établi des écoles tout d'abord en Italie et puis ailleurs dans le monde. Florence Nightingale a changé la formation en science infirmière et le domaine de la santé au Royaume-Uni ainsi que dans d'autres pays. II ne faut toutefois pas assimiler entrepreneur social et entreprise sociale : une entreprise sociale n'est pas forcément dirigée par un entrepreneur social. Par exemple, les multiples filiales ou co-entreprises de la Grameen Bank ne sont pas forcément dirigées par des personnes présentant les caractéristiques de l'entrepreneur social.

Quant à l'entreprise sociale, on peut la représenter comme un système social avec une interprétation très large ou comme une entité incluant la notion de commerce (Briand, 2001). Ainsi, Ménard (2004) exclut les entités à caractère non marchand, telle une association philanthropique, de la définition d'une entreprise. Favreau (2006) propose d'ailleurs une typologie de trois grandes familles d'organisations de l'économie sociale, soit les associations, les coopératives et les mutuelles. Pour notre part, l'entreprise sociale est interprétée de manière très large et peut se définir tout simplement comme une organisation qui vise principalement des objectifs sociaux. On peut alors répartir les différentes activités de ces entreprises sociales sur un axe allant de l'offre de produits ou services d'intérêt " public " ou " général » qui servent les intérêts de toute une population donnée, à l'offre de produits ou services d'intérêt " commun » ou " collectif » (Painter, 2006) visant à répondre aux intérêts de seulement un groupe donné de membres ou de participants. Un exemple d'intérêt général pourrait être une soupe populaire ou une entreprise qui favorise l'insertion sur le marché du travail alors qu'un exemple d'intérêt collectif pourrait être une association sportive organisant les activités de hockey des enfants d'une localité ou d'une coopérative de travailleurs forestiers.

Les entreprises sociales peuvent prendre différentes formes et il est possible de les distinguer selon différentes caractéristiques (Alter, 2006; Crossan, Bell et Ibbotson, 2003). Pour mieux comprendre la diversité des formes que revêtent ces organismes, il est possible d'examiner les types d'entreprises. II y a les entreprises gouvernementales, comme les départements, les 
agences et les sociétés de la Couronne et les entreprises paragouvernementales qui, tout en conservant une certaine autonomie, sont quand même dépendantes des ressources de l'État, comme les hôpitaux et les universités. Lorsqu'il est question d'économie sociale, la mention du secteur sans but lucratif et bénévole est aussi inévitable et très liée (Valéau, Cimper et Filion, 2004). Pour certains, la majorité des entreprises sociales sont des organismes sans but lucratif (OSBL). Un organisme sans but lucratif est une " entité qui n'a normalement pas de titres de propriété transférables et dont l'organisation et le fonctionnement visent exclusivement des fins sociales, éducatives, professionnelles, religieuses, charitables, ou de santé, ou toute autre fin à caractère non lucratif " (Institut Canadien des Comptables Agréés, 2009, paragraphe 4400.02a). Par opposition, une entreprise à but lucratif est une "entité établie dans le but de réaliser des profits et dont les titres de capital ou autres droits de propriété sont généralement transférables et susceptibles de procurer un profit à son propriétaire exploitant, ses associés ou ses actionnaires, ou de leur occasionner une perte » (Ménard, 2004, p.935). Les entreprises hybrides se situent entre l'entreprise sans but lucratif et l'entreprise à but lucratif, ayant des visées à la fois philanthropiques et commerciales qui peuvent emprunter des caractéristiques de chacune. Une coopérative d'alimentation représente un exemple d'entreprise hybride.

Afin de mieux comprendre le concept, il peut être utile de présenter les entreprises sociales en comparant les rôles social et commercial (Austin, Stevenson et Wei-Skillern, 2006). Comme l'illustre le Tableau B, les entreprises sociales peuvent être comparées à des entreprises socialement responsables, à des entreprises socialement neutres et à des entreprises socialement irresponsables.

Tableau B - Comparaison des rôles sociaux et commerciaux des entreprises

\begin{tabular}{|c|c|c|c|}
\hline \multirow[t]{2}{*}{ Type d'entreprise } & \multirow[t]{2}{*}{ Rôle social } & \multicolumn{2}{|c|}{ Rôle commercial } \\
\hline & & $\begin{array}{c}\text { Échanges } \\
\text { commerciaux }\end{array}$ & $\begin{array}{c}\text { Répartition des bénéfices } \\
\text { commerciaux }\end{array}$ \\
\hline \multirow{3}{*}{ Entreprise sociale } & \multirow{2}{*}{$\begin{array}{l}\text { Rôle } \\
\text { exclusivement } \\
\text { social }\end{array}$} & $\begin{array}{c}\text { Aucun échange } \\
\text { commercial }\end{array}$ & $\mathrm{S} / \mathrm{O}$ \\
\hline & & \multirow{5}{*}{$\begin{array}{l}\text { Présence } \\
\text { d'échanges } \\
\text { commerciaux }\end{array}$} & $\begin{array}{l}\text { Bénéfices répartis à 100\% } \\
\text { vers le social }\end{array}$ \\
\hline & $\begin{array}{l}\text { Rôle social } \\
\text { prioritaire }\end{array}$ & & $\begin{array}{c}\text { Bénéfices répartis } \\
\text { majoritairement vers le social }\end{array}$ \\
\hline $\begin{array}{l}\text { Entreprise } \\
\text { socialement } \\
\text { responsable }\end{array}$ & $\begin{array}{l}\text { Rôle social } \\
\text { minoritaire }\end{array}$ & & $\begin{array}{c}\text { Bénéfices répartis } \\
\text { minoritairement vers le social } \\
\text { et majoritairement aux } \\
\text { actionnaires }\end{array}$ \\
\hline $\begin{array}{c}\text { Entreprise } \\
\text { socialement } \\
\text { neutre } \\
\end{array}$ & $\begin{array}{l}\text { Aucun rôle } \\
\text { social }\end{array}$ & & $\begin{array}{c}\text { Bénéfices répartis à } 100 \% \text { aux } \\
\text { actionnaires }\end{array}$ \\
\hline $\begin{array}{c}\text { Entreprise } \\
\text { socialement } \\
\text { irresponsable } \\
\end{array}$ & $\begin{array}{l}\text { Rôle social } \\
\text { négatif }\end{array}$ & & $\begin{array}{l}\text { Bénéfices répartis à } 100 \% \text { aux } \\
\text { actionnaires }\end{array}$ \\
\hline
\end{tabular}

Le rôle commercial évoqué dans le Tableau B comprend deux dimensions, soit la présence d'échanges commerciaux et la répartition des bénéfices commerciaux. L'entreprise sociale doit accorder un rôle exclusif ou prioritaire au rôle social. Le rôle économique est secondaire. 
Il peut ou non y avoir des échanges commerciaux. Au niveau de la répartition des bénéfices commerciaux, les entreprises sociales répartiront totalement ou majoritairement les bénéfices vers le social plutôt qu'aux actionnaires.

La colonne relative au rôle social permet de mettre en évidence le niveau d'engagement social des différentes catégories d'entreprises comparées dans le tableau $B$. Ce critère du rôle social fait inévitablement référence à la notion de responsabilité sociale de l'entreprise (RSE), définie comme "l'intégration volontaire par les entreprises de préoccupations sociales et environnementales à leurs activités commerciales et leurs relations avec leurs parties prenantes » (European Commission, 2001). La diffusion mondiale de ce concept a donné lieu à des modes d'appropriation que Capron qualifie pudiquement de "graduées " (Capron, 2009 , p. 2). Tout d'abord parce que la RSE inclut de nombreuses problématiques, telles que les droits humains et ceux des travailleurs, l'environnement, l'éthique, etc., et que les entreprises s'impliquent à des degrés divers dans chacune d'entre elles. Mais surtout parce que les actions des entreprises couvrent toute une palette, allant d'un engagement réel, sincère et efficace, à des pratiques de détournement qualifiées de greenwashing (" mascarade écologique ») ou de socialwashing. Ces dernières consistent à utiliser les thèmes de la RSE comme arguments de communication destinés à améliorer la réputation de l'entreprise sans réelle modification du comportement de celle-ci.

Trois conceptions de la RSE coexistent (Capron, 2009): une conception plus ou moins traditionnelle et paternaliste qui conduit, par exemple, à des actions philanthropiques; une conception plus utilitariste, encouragée notamment par la Commission européenne, qui repose sur l'idée que la RSE a des retombées économiques positives; enfin, une conception plus citoyenne, basée sur l'idée d'un encastrement de l'entreprise dans la société, qui rendrait la firme nécessairement redevable à la société. Cependant, aucune de ces conceptions n'implique que le volet social doit primer sur le volet économique. II s'agit davantage pour l'entreprise, même dans la dernière des trois conceptions, d'assumer pleinement les conséquences sociales ou environnementales de son activité. L'idée de la RSE est davantage de faire un profit tout en faisant le bien (ou sans faire de mal), que de faire du bien tout en faisant un profit.

Si l'on revient aux différentes catégories d'entreprises présentées dans le Tableau B, une entreprise sociale n'est donc pas une entreprise qui a une politique de RSE, même sincère: elle va au-delà, en faisant de sa mission sociale une priorité. Une entreprise socialement responsable serait, toujours au sens du tableau $B$, une entreprise qui ne place pas le rôle social au premier plan mais est tout de même attentive aux besoins de la société et à son rôle dans celle-ci. En ce sens, ce serait une entreprise dont le rôle est prioritairement économique, mais appliquant effectivement les principes de la RSE à des degrés divers. En revanche, une entreprise pratiquant le socialwashing pourrait relever de la catégorie " entreprise neutre » (rôle social ni positif ni négatif), voire " entreprise irresponsable », si elle se dit responsable tout en ayant un impact social négatif.

Pour illustrer le concept d'entreprise sociale telle que définie dans le Tableau B, on peut citer Ashoka, Grameen Bank au Bangladesh, Bangladesh Rural Advancement Committee, Green Belt Movement au Kenya, Highlander Research and Education Center aux États-Unis, Plan Puebla au Mexique et Self-Employed Women's Association en Inde (Alvord, Brown et Letts,2004). Au Canada, Johnson (2003) a répertorié, notamment, Meal Exchange à Waterloo, Santropol Roulant à Montréal, Generation Solar à Peterborough, Home Grown Organic Food à Halifax, Youth One à Edmonton et Humanity Link à Toronto. D'autres 
exemples intéressants sont les Centres de pédiatrie sociale du Dr Gilles Julien, le restaurant Robin des Bois à Montréal, Communauto et Virtuocar.

Ces précisions sur les différents concepts liés à l'entrepreneuriat social montrent que ce phénomène repose à la fois sur des personnes et des organisations. La section suivante s'attache à montrer que ces entrepreneurs sociaux ont choisi une forme d'engagement qui relève de la participation citoyenne dont le lieu privilégié d'expression est l'entreprise sociale.

\section{CONTRIBUTION DE L'ENTREPRENEURIAT SOCIAL À LA PARTICIAPATION CITOYENNE}

Il convient de noter tout d'abord que la participation citoyenne est un concept large et difficile à définir puisqu'il peut y avoir différentes significations selon les disciplines. Par exemple, on le retrouve en sciences politiques comme un sous-concept de la participation publique qui désigne la participation des citoyens aux dispositifs institués par l'État (Bherer, 2006), ainsi qu'en sociologie dans les études s'intéressant à la participation des individus à la définition et au changement de leur société par des actions concrètes et non par un simple engagement émotionnel (Couton et Gaudet, 2008). La participation sociale fait aussi l'objet d'études en anthropologie qui abordent les diverses formes de citoyenneté selon les contextes culturels (Paley, 2002).

Une définition très classique de la participation citoyenne est donnée par Arnstein, selon lequel il s'agit d' " une expression radicale qui désigne le pouvoir des citoyens » (CES, 2006, p.1). Selon nous, afin de mieux saisir le concept de la participation citoyenne, il convient de préciser les dimensions de la citoyenneté et les différentes actions que les citoyens peuvent entreprendre pour l'exprimer. Selon Lemieux (2004), la citoyenneté représente " un terrain délimité par trois bornes de nature civique, politique et sociale respectivement ". Ce même auteur soutient qu'à chacune de ces bornes correspond une dimension de la participation citoyenne. II s'agit de : (1) la délibération démocratique qui consiste à prendre part dans les débats sur les enjeux sociaux, politiques et économiques; (2) la participation politique qui consiste pour les citoyens d'exercer les droits politiques qui leurs sont conférés, dont le plus important est le droit de vote, et (3) la participation à la société civile qui consiste dans l'implication des citoyens non pas dans les partis politiques ou les instances gouvernementales mais plutôt dans des organisations (telles que les entreprises sociales et les associations) dont l'objectif est de défendre et de faire connaître des droits sociaux.

\section{Tableau C - Les huit échelons sur l'échelle de participation d'Arnstein}

\begin{tabular}{|c|c|c|}
\hline & Échelons de participation & Niveau de participation \\
\hline 8 & Contrôle citoyen & \multirow{3}{*}{ Pouvoir effectif des citoyens } \\
\hline 7 & Délégation de pouvoir & \\
\hline 6 & Partenariat & \\
\hline 5 & Rassurance & \multirow{3}{*}{ Coopération symbolique } \\
\hline 4 & Consultation & \\
\hline 3 & Information & \\
\hline 2 & Thérapie & \multirow{2}{*}{ Non participation } \\
\hline 1 & Manipulation & \\
\hline
\end{tabular}


La participation citoyenne va au-delà d'un simple engagement émotionnel pour se manifester par des efforts, actes et gestes concrets et visibles qui sont adoptés par les citoyens. Dans ce cadre, Arnstein (1969) définit huit niveaux de participation citoyenne (voir le Tableau $C$ en lisant à partir du bas) qui vont de la non participation à la participation correspondant à un pouvoir effectif des citoyens. Même si nous convenons avec l'auteur que cette échelle est simplificatrice, elle comporte une utilité pour faciliter la compréhension des gestes posés et pour situer l'ensemble des actions, incluant l'entrepreneuriat social.

Les deux niveaux inférieurs correspondent à des exemples de non participation. Les détenteurs du pouvoir accordent un semblant de participation aux citoyens, sans leur donner le véritable pouvoir de changer les choses. Le cas de la " manipulation " ressemble à un exercice de relations publiques où l'on influence et instrumentalise le soutien des citoyens. Par exemple, ces derniers sont invités à appartenir à un comité consultatif qui va les conduire à apposer un sceau sur des projets en fait déjà validés. Dans le cas de la " thérapie ", l'artifice consiste à impliquer les citoyens dans un processus qui tend à leur faire accepter leur situation, voire à les culpabiliser par rapport à elle, tout en leur faisant croire qu'on cherche à agir avec eux pour résoudre le problème.

Les trois niveaux du milieu correspondent à des exemples de coopération symbolique. Ainsi, les citoyens ont un certain accès à l'information et peuvent se faire entendre, sans pour autant avoir de véritable pouvoir de décision. L' information " des citoyens face à leurs droits, responsabilités et options est une étape importante de leur participation. Elle ne doit toutefois pas se limiter à une communication à sens unique, qui ne laisse pas place à la négociation. Par exemple, il peut s'agir de distribuer des dépliants avec certaines informations choisies ou d'organiser une réunion avec de l'information superficielle. Solliciter l'avis des citoyens lors d'une " consultation » est aussi un processus intéressant. Encore faut-il qu'il y ait prise en compte des préoccupations et idées émises. Par exemple, il peut s'agir d'audiences publiques, d'enquête d'opinions ou de réunions de quartier. Dans le cas de la " rassurance », il y a une certaine influence du public, qui reste toutefois très limitée. Par exemple, la nomination d'un nombre insuffisant de représentants socioéconomiques triés sur le volet dans des comités permet à ceux qui détiennent le pouvoir de conserver la majorité du pouvoir décisionnel.

Les trois niveaux supérieurs correspondent à des exemples de pouvoir effectif des citoyens. II y a donc une négociation et un échange avec les détenteurs du pouvoir. Le " partenariat » permet une redistribution du pouvoir. Par exemple, il peut s'agir de la participation à des comités où il y a un réel partage des responsabilités et des décisions entre les citoyens et les détenteurs du pouvoir. La " délégation de pouvoir " assure que les citoyens soient munis de moyens suffisants pour exercer une influence réelle et même dominer le pouvoir décisionnel. Enfin, le " contrôle citoyen " correspond à un niveau de participation où les citoyens détiennent le contrôle véritable des décisions, s'ils le désirent. Par exemple, la nomination d'un nombre majoritaire de représentants dans des comités permet à ceux-ci d'exercer pleinement le pouvoir décisionnel. Ainsi, seuls les niveaux les plus élevés (partenariat, délégation de pouvoir et contrôle citoyen) correspondent à une participation citoyenne effective, c'est-à-dire à un véritable pouvoir de décision, voire de gestion.

Tout en nous concentrant sur ces trois derniers niveaux de l'échelle d'Arnstein (pouvoir effectif des citoyens), nous soutenons que la participation citoyenne effective correspond à l'ensemble des actions par lesquelles les citoyens participent à l'espace public et dans la société et ce, suite à des initiatives pouvant provenir de trois sources différentes, soit : l'État, 
les regroupements de la société civile et les citoyens eux-mêmes. Le Tableau D présente des exemples d'initiatives prises par ces trois sources.

\section{Tableau D - Exemples d'initiatives de participation citoyenne}

\begin{tabular}{|c|c|c|c|c|}
\hline Initiateur & Etat & Regroupements de & \multicolumn{2}{|c|}{ Citoyens } \\
\hline \multirow[t]{2}{*}{$\begin{array}{l}\text { Exemples } \\
\text { d'initiatives }\end{array}$} & \multirow{2}{*}{$\begin{array}{l}\text { - élections } \\
\text { - assemblée } \\
\text { citoyenne } \\
\text { - conseil de } \\
\text { quartier } \\
\text { - comité } \\
\text { d'usager } \\
\text { dans les } \\
\text { hôpitaux } \\
\text { - audience } \\
\text { publique }\end{array}$} & \multirow{2}{*}{$\begin{array}{l}\text { - activités d'une } \\
\text { association } \\
\text { communautaire } \\
\text { (manifestation, } \\
\text { lobbying) } \\
\text { - opération de } \\
\text { soutien à des } \\
\text { réfugiés } \\
\text { organisée par un } \\
\text { parti politique } \\
\text { - opération de } \\
\text { défense d'un } \\
\text { bassin d'emploi } \\
\text { organisée par un } \\
\text { syndicat }\end{array}$} & $\begin{array}{l}\text { - création d'une } \\
\text { entreprise avec une } \\
\text { mission sociale } \\
\text { - travail dans une } \\
\text { entreprise sociale } \\
\text { - bénévolat dans une } \\
\text { entreprise sociale } \\
\text { - achat auprès d'une } \\
\text { entreprise sociale }\end{array}$ & $\begin{array}{l}\text { Occasions de } \\
\text { participation } \\
\text { citoyenne créées } \\
\text { au sein des } \\
\text { entreprises } \\
\text { sociales }\end{array}$ \\
\hline & & & $\begin{array}{l}\text { - engagement } \\
\text { communautaire } \\
\text { - membre d'une } \\
\text { coopérative de } \\
\text { travailleurs } \\
\text { - opinion du lecteur } \\
\text { dans les journaux }\end{array}$ & \\
\hline
\end{tabular}

Premièrement, il y a les initiatives de l'État. La participation des citoyens aux élections par le biais du vote ou à une audience publique organisée par le gouvernement sont des exemples de participation citoyenne à des initiatives de l'État. L'Organisation de coopération et de développement économiques atteste dans son rapport Des citoyens partenaires (OCDE, 2001) que l'implication des citoyens dans la prise de décision publique est un élément de la bonne gouvernance. D'ailleurs, le Canada a augmenté le nombre de forums et de débats publics officiels ces dernières années (Bherer, 2006). Dans ce cadre, on peut citer des assemblées des citoyens en Colombie-Britannique et en Ontario (Warren, 2008) ainsi que les deux consultations publiques au Québec sur les accommodements raisonnables et la condition de vie des aînés. II semble ainsi qu'on témoigne d'un véritable virage participatif qui reconnaît de plus en plus aux citoyens un droit de regard sur l'action des gouvernements et les incite à prendre part dans le processus de décision publique (Blatrix, 2002).

Deuxièmement, il y a les initiatives des regroupements de la société civile. Ainsi, divers groupes (organismes communautaires, partis politiques, syndicats) s'impliquent dans les débats de société en organisant des manifestations ou des programmes. À l'automne 2009, la revitalisation du Parc Lansdowne à Ottawa a suscité de vifs débats autour d'un projet de partenariat entre un groupe d'entrepreneurs et le Conseil municipal. Dans ce cadre, l'Association des résidents du quartier Glebe, où se trouve le parc en question près du canal Rideau, a été particulièrement active pour s'opposer au projet qui a néanmoins reçu l'aval du Conseil municipal.

Troisièmement, les citoyens eux-mêmes peuvent amener des initiatives visant à rendre service à la société et à participer à la définition du collectif. Dans ce groupe d'initiatives, nous distinguons tout d'abord les initiatives centrées principalement autour de l'entrepreneuriat social et des entreprises sociales. Notons la création d'une entreprise avec une mission 
sociale, le travail dans une entreprise sociale, l'adhésion à une coopérative de travailleurs, le bénévolat dans des entreprises sociales et l'achat auprès d'une entreprise sociale.

En résumé, l'entrepreneuriat social se situe tout d'abord dans la troisième dimension de la participation citoyenne (à savoir la participation à la société civile), aux trois niveaux supérieurs de l'échelle d'Arnstein (pouvoir effectif des citoyens) et résulte des initiatives entreprises par les citoyens eux-mêmes. Les entrepreneurs sociaux sont, en effet, des individus qui essaient de répondre à des besoins sociaux spécifiques en proposant des solutions innovatrices et en impliquant d'autres personnes dans leur démarche. L'entrepreneuriat social, en tant qu'ensemble d'activités et de processus permettant de créer et maintenir de la valeur sociale, constitue un cadre dans lequel des individus peuvent soutenir ou participer à un système de gouvernance dont les principaux objectifs sont sociaux et non politiques (au sens de système de décision politique) ou principalement économiques (bien que des activités économiques puissent être menées par une entreprise sociale). En agissant dans le cadre d'une entreprise sociale, l'entrepreneur social donne l'occasion à d'autres citoyens d'exprimer leur citoyenneté, en leur permettant par exemple de travailler bénévolement dans l'entreprise sociale ou en leur vendant ses produits et services dans le principal but d'obtenir leur soutien dans son parcours.

Dans ce cadre, l'entrepreneur social constitue un archétype de citoyen qui décide d'utiliser une démarche entrepreneuriale pour répondre à des besoins sociaux. Loin de participer symboliquement au changement social, il décide d'agir de façon innovante et se donne le pouvoir de s'aider soi-même et d'aider les autres. Le lieu privilégié de ce type de participation citoyenne est alors l'entreprise sociale, qui va constituer l'organisation (et son système de gouvernance) par laquelle des citoyens peuvent agir plus ou moins directement sur la société. Cette participation peut prendre plusieurs formes : l'entrepreneur " créateur » de l'entreprise sociale a déjà été évoqué ci-dessus, mais il n'est pas le seul à " participer » à la société par le truchement de l'entreprise sociale. On peut en effet analyser le rôle des salariés, bénévoles et des éventuels clients d'une entreprise sociale comme une participation citoyenne plus ou moins directe. À des titres divers, ils sont des partenaires de l'entreprise sociale; ils lui fournissent des ressources, et peuvent, selon les cas, participer à la conduite de l'entreprise, ou l'influencer en tant que parties prenantes.

Pour illustrer notre propos, citons quelques exemples relevant de cette catégorie. Tout d'abord, un exemple permet de lier l'entreprise sociale et la participation citoyenne d'une manière très directe. À ce propos, le parcours de Saïd Hammouche, créateur de Mozaïk RH (cabinet de recrutement spécialisé dans le placement de jeunes issus de la diversité et des quartiers difficiles), est pertinent. II a choisi de saisir l'occasion que représentait la mauvaise prise en compte de ce besoin social par le secteur public en France pour proposer une offre professionnelle originale venant satisfaire une demande bien réelle (Mozaïk $\mathrm{RH}$, s.d.). Saïd Hammouche participe à la résolution d'un problème de société en assumant le contrôle réel de son activité sociale.

Situé à Montréal, Robin des Bois est un restaurant à but non lucratif où quelques employés sont soutenus par des bénévoles (Robin des Bois, s.d.). Tous les profits réalisés par les ventes de repas et de produits sont redistribués à quelques organismes de bienfaisance choisis qui œuvrent dans la communauté montréalaise afin de vaincre la solitude, l'isolement et la pauvreté. Les six organismes choisis par Robin des Bois pour bénéficier de leur soutien sont : le Chaînon, le Refuge des jeunes, Chez Doris, Jeunesse au soleil, Santropol Roulant et Cactus Montréal. Les trois premiers sont des refuges pour femmes ou jeunes en difficulté, tandis que les trois suivants sont : un centre communautaire, un service de popote roulante et 
un service d'intervention au niveau des drogues et des travailleuses du sexe. II s'agit d'une occasion d'effectuer du bénévolat d'une manière créative et séduisante, en cuisine ou en salle. Tant les bénévoles que les clients sont invités à une prise de conscience et à une responsabilisation individuelle et collective envers les personnes démunies. Bien que seules les fondatrices du restaurant entrent dans le groupe des entrepreneurs sociaux, le fait d'y travailler ou d'y faire du bénévolat représente tout de même une forme de participation citoyenne, puisque l'on participe à une entreprise qui elle-même s'implique activement dans la vie de la communauté. (Pour les bénévoles, il s'agit d'une forme de participation moins forte, mais ils participent tout de même, un peu comme lorsqu'on est client d'une entreprise sociale.) Notre thèse est que l'entreprise sociale permet des degrés divers de participation citoyenne : forte (entrepreneuriat social) ou plus faible (soutien de l'entreprise sociale par la consommation ou l'engagement dans un emploi qui implique souvent une rémunération plus faible que dans une entreprise classique mais contribue au développement social).

De son côté, Pro Mujer est maintenant une organisation internationale qui a été initialement fondée en Bolivie en 1990 par deux femmes, Lynn Patterson et Carmen Valesco, et qui a par la suite exporté ses activités au Nicaragua, au Pérou et au Mexique (http:www.promujer.org). Son objectif est de fournir aux femmes rurales très démunies des microcrédits qui les aident à financer différents types de microprojets, mais surtout de les éduquer en leur fournissant des ateliers de formation en gestion des affaires et de l'information sur l'importance de scolariser leurs enfants et de prêter attention à la santé de leurs familles. Ces clientes étant très pauvres, elles n'ont pas accès au crédit des grandes institutions financières mais peuvent avoir des microcrédits de Pro Mujer allant de 50 \$à 300 \$. Des taux d'intérêt raisonnables leur sont imposés de façon à ne pas compromettre la viabilité de leurs projets. Étant donné le volume des affaires, le montant total d'intérêt recueilli permet à Pro Mujer de couvrir ses coûts financiers et opérationnels ainsi que d'absorber les pertes dues aux défauts de paiement. Ainsi, les deux principales fondatrices de Pro Mujer sont des entrepreneures sociales qui ont pu outiller financièrement et intellectuellement des millions de femmes qui n'avaient même pas de quoi nourrir leurs familles pour qu'elles soient de meilleures mères de famille et des entrepreneures éduquées et capables. Selon une enquête réalisée, ces femmes ont pu doubler leurs revenus après seulement deux ans de participation au programme offert par Pro Mujer (Alter, 2006).

Ces développements permettent ainsi de mettre en lumière le rôle que jouent les entrepreneurs sociaux pour se donner eux-mêmes une véritable occasion de participation citoyenne et faciliter en même temps celle des autres. En effet, en créant des entreprises sociales, ils participent à la société civile par la mise en œuvre de solutions innovantes à des problèmes sociaux donnés et mobilisent leurs concitoyens en leur donnant diverses possibilités de contribution à l'entreprise sociale et de participation citoyenne. Ils créent des emplois pour des personnes ayant des difficultés particulières d'insertion sur le marché du travail (personnes non qualifiées, handicapés, ex-détenus, minorités ethniques), développent des services sociaux, et génèrent des revenus pour financer leurs activités sociales. 
Brouard, Larivet, et Sakka (2010)

\section{CONCLUSION}

L'objectif de la présente contribution, encore au stade exploratoire, était d'apporter un certain éclairage du lien qui existe entre l'entrepreneuriat social et la participation citoyenne. Pour ce faire, nous avons commencé par expliquer le contexte pour le développement de l'entrepreneuriat social, suivi d'une définition de ce concept et de notions connexes telles qu' "entreprise sociale » et "économie sociale». Nous avons ainsi souligné que l'entrepreneuriat social était un concept émergeant, mal connu et défini de façon non consensuelle. Sans nul doute, il s'agit d'un phénomène prometteur dans l'atteinte d'objectifs sociaux et de développement économique local (Brinckerhoff, 2000). Ce phénomène est particulièrement important dans une société dont les besoins sociaux ne cessent de s'accroître mais dont les ressources sont limitées. Les entrepreneurs sociaux sont des individus qui adoptent des actions concrètes visant un véritable changement social, quelle que soit son échelle (locale ou limitée dans le nombre de personnes qu'il touche), et ils ne se contentent pas d'un simple engagement émotionnel envers une problématique sociétale donnée.

Afin de faire le lien entre l'entrepreneuriat social et la participation citoyenne, nous avons précisé, dans la troisième section de l'article, que ce dernier concept présente trois dimensions, à savoir la délibération démocratique, la participation politique et la participation à la société civile, et que l'entrepreneuriat social se situe au niveau de cette dernière dimension. La troisième section soutient aussi que la participation citoyenne peut se manifester à des degrés différents allant de la non-participation au pouvoir effectif des citoyens et que l'entrepreneuriat social se situe aux niveaux les plus élevés de la participation citoyenne puisqu'il correspond à des actions concrètes et des efforts tangibles adoptés par les entrepreneurs sociaux pour résoudre des problèmes sociaux et participer au changement de leur société.

Nous avons également spécifié que la participation citoyenne peut être le résultat d'initiatives entreprises par l'État, par les regroupements de la société civile ou par les citoyens euxmêmes et que l'entrepreneuriat social correspond à une initiative de la part des citoyens (entrepreneurs sociaux) afin de mobiliser d'autres citoyens pour une cause sociale donnée dans ce lieu privilégié qu'est l'entreprise sociale. Ainsi, par la création d'entreprises sociales, les entrepreneurs sociaux expriment une participation citoyenne d'un niveau très élevé, tout en permettant à certains de leurs concitoyens de manifester eux aussi un niveau plus ou moins élevé de participation citoyenne. Les citoyens clients, les citoyens salariés ou les citoyens bénévoles liés à ces entreprises sociales participent à assurer la viabilité de l'entreprise sociale. Le pouvoir qu'ils donnent aux entreprises sociales grâce aux ressources qu'ils fournissent est une forme de participation qui peut s'avérer puissante.

Plusieurs défis sont mentionnés par Johnson (2000; 2003) afin de développer le concept d'entrepreneuriat social. Compte tenu de son émergence, le support aux entrepreneurs sociaux ne fait pas encore totalement partie des programmes actuels d'aide aux entrepreneurs. Il y a donc lieu pour les décideurs institutionnels d'ajouter ce type d'entrepreneur dans la liste des entrepreneurs à aider tout en portant une attention particulière à la spécificité qui leur est propre. Contrairement à ce qui se passe aux États-Unis, par exemple, la formation en entrepreneuriat social est quasi inexistante au Canada. II existe cependant certains programmes de formation continue touchant à ce type d'entrepreneuriat. II y a lieu en outre de développer l'entrepreneuriat social dans les universités. Pour développer une culture entrepreneuriale et ainsi prospérer, il est nécessaire de mieux former et de mieux 
informer les différents intervenants, que ce soit les gouvernements, les élus locaux, les éducateurs et la population en général (Fortin, 2002).

La contribution principale de cet article consiste ainsi à explorer le rôle que peut jouer l'entrepreneuriat social dans le renforcement de la participation citoyenne en essayant de clarifier la zone de chevauchement qui existe entre ces deux concepts. Des recherches futures pourront aller de l'avant pour préciser davantage ce lien mais aussi pour clarifier la différence entre ces deux concepts en expliquant, par exemple, les actions qui peuvent relever de l'entrepreneuriat social mais qui n'ont aucun lien avec la participation citoyenne. Espérons que cette contribution permettra d'accroître l'intérêt pour l'entrepreneuriat social et ses impacts dans la société.

\section{BIBLIOGRAPHIE}

Alter, K. (2006). Social Enterprise Typology. Consulté le 13 avril 2009 à http://www.virtueventures.com/setypology.pdf

Alvord, S, Brown, D et Letts, C (2004). Social entrepreneurship and societal transformation. Journal of Applied Behavioral Science, 40(3), 260-282.

AMAP (s.d.). Site national des AMAP. Qu'est-ce qu'une AMAP. Pourquoi participer ? Consulté le 9 août 2009 à http://www.reseau-amap.org/amap.php

Anderson, R.B., Dana, L.P. et Dana, T.E. (2006). Indigenous land rights, entrepreneurship, and economic development in Canada: "Opting-in" to the global economy. Journal of World Business, 41(1), 45-55.

Arnstein, S.R. (1969). A Ladder of Citizen Participation. Journal of the American Institute of Planners, 35(4), 216-224.

Arts Quarter (2009). Impacts of the recession on the UK cultural and not-for-profit sectors - Findings from the Arts Quarter survey. London : Arts Quarter LLP.

Audet, J. et Julien, P.A. (2006). L'entrepreneuriat social au Québec. L'exemple des centres de formation en entreprise et récupération, Recherches sociographiques, XLVII(1), 69-94.

Austin, J., Stevenson, H. et Wei-Skillern, J. (2006). Social and commercial entrepreneurship: Same, different, or both?, Entrepreneurship: Theory \& Practice, 30(1), 1-22.

Bacq, S. et Janssen, F. (2008a). From social entrepreneurship as a practice to legitimate field of research: literature review and classification. Louvain School of Management. Center for Research in Entrepreneurial Change \& Innovation Strategies. Working paper 06/2008.

Bacq, S. et Janssen, F. (2008b). Définition de l'entrepreneuriat social : Revue de la littérature selon les critères géographiques et thématiques. Actes du $9^{\text {ème }}$ CIFEPME. 28-31 octobre, Louvain-la-Neuve, Belgique.

Barendsen, L. et Gardner, H. (2004). Is the social entrepreneur a new type of leader? Leader to Leader, 2004(34), 43-50.

Bherer, L. (2006). La démocratie participative et la qualification citoyenne : À la frontière de la société civile et de l'État. Nouvelles pratiques sociales, 18(2), 24-38. 
Blatrix, C. (2002). Devoir débattre. Les effets de l'institutionnalisation de la participation sur les formes de l'action collective. Politix. Revue des sciences sociales du politique, 15(57), 79-102.

Bornstein, D. (2007). How to change the world: Social entrepreneurs and the power of new ideas. New York : Oxford University Press.

Boucher, J., Favreau, L., Guindon, G. et Hurtubise, L. (2000). Développement local, organisation communautaire et économie sociale. Une recension des écrits (1990-2000). Université du Québec à Hull, Chaire de recherche en développement communautaire (CRDC), document de travail, automne.

Briand, L. (2001), Analyse structurationniste du contrôle de gestion dans la modernité et la modernité avancée. Thèse de doctorat en administration, HEC Montréal.

Brinckerhoff, P. (2000). Social entrepreneurship: The art of mission-based venture development. New York : John Wiley \& Sons.

Brouard, F. et Larivet, S. (2009), Social entrepreneurship: Definitions and boundaries, Conférence 2009 ANSER-ARES, 27-29 mai, Ottawa, Canada.

Capron, M. (2009), La responsabilité sociale d'entreprise, in L'Encyclopédie du développement durable, 99. Paris : Éditions des Récollets. Consulté le 9 septembre 2010 à http://encyclopediedd.org/IMG/pdf_N_99_Capron.pdf CES (Comité d'évaluation et de suivi) (2006). Une échelle de participation citoyenne - S. Arnstein. Consulté le 9 août 2009 à

http://www.anru.fr/IMG/pdf/Echelle_de_participation_citoyenne_Sherry_Arnstein.pdf

Christie, M.J. et Honig, B. (2006). Social entrepreneurship: New research findings. Journal of World Business, 41(1), 1-5.

Couton P. et Gaudet S. (2008). Rethinking social Participation: The case of immigrants in Canada. Journal of International Migration and Integration, 9(1), 21-45.

Crossan, D., Bell, J. et Ibbotson, P. (2003). Towards a classification framework for social enterprises. Working paper, University of Ulster.

Cutt, J. et Murray, V.V. (2000). Accountability and effectiveness evaluation in non-profit organizations. London : Routledge.

Davis, S. (2002). L'entrepreneuriat social : Vers une culture entrepreneuriale propice au développement économique et social. Actes du Sommet de l'emploi des jeunes, septembre, 7-11.

Dees, G. (1998). Enterprising nonprofits. Harvard Business Review, 76(1), 55-65.

Edwards, M. (2008). Just another emperor? The myths and realities of philanthrocapitalism, New York: Demos/Young Foundation.

Elkington, J. et Hartigan, P. (2008). The Power of unreasonable people: How social entrepreneurs create markets that change the world. Boston : Harvard Business School Press.

European Commission (2001). Green paper: Promoting a European framework for corporate social responsibility. Brussels : Commission of the European Communities.

Favreau, L. (2005). Qu'est que l'économie sociale? Synthèse introductive. Université du Québec en Outaouais, Chaire de recherche du Canada en développement des collectivités (CRDC). Série : Recherche. No. 35, mars. 
Favreau, L. (2006), Économie sociale et politiques publiques - L’expérience québécoise. Horizons, 8(2), 7-15.

Foot, D.K. et Stoffman, D. (1996). Entre le boom et l'écho, Montréal : Boréal.

Fortin, P.A. (2002). La culture entrepreneuriale : un antidote à la pauvreté, Montréal / Charlesbourg : Éditions Transcontinental/Éditions de la Fondation de l'entrepreneurship.

Guay, M.H., et Riverin, N. (2006). L'entrepreneuriat social au Québec. Bulletin CVRCE. No 88.

Haugh, H. (2007). Community-led social venture creation. Entrepreneurship: Theory \& Practice, 31(2), 161-182.

Institut Canadien des Comptables Agréés (2009), Manuel de I'ICCA. Toronto : ICCA. Johnson, S. (2000). Literature review on Social entrepreneurship. Consulté le 17 novembre 2008 à http://www.bus.ualberta.ca/ccse/whatis

Johnson, S. (2003). Young social entrepreneurs in Canada. Consulté le 21 mai 2009 à http://www.socialinnovationexchange.org/node/105

Joyal, A. (1999). Économie sociale : le bilan québécois. Montréal : L'île de la tortue.

Lemieux, G. (2004). Remixer la cité. La participation citoyenne des jeunes québécois issus des minorités visibles. Conseil permanent de la jeunesse et Conseil des relations interculturelles. Rapport de recherche, 126 p. http://collections.banq.qc.ca/ark:/52327/bs48836 (consulté le 19 septembre, 2010).

Mair, J. et Marti, I. (2006). Social entrepreneurship research: A source of explanation, prediction, and delight. Journal of World Business, 41(1), 36-44.

Ménard, L. (2004). Dictionnaire de la comptabilité et de la gestion financière, $2^{\mathrm{e}}$ éd., Montréal : Institut Canadien des Comptables Agréés.

Mozaïk RH (s.d.). Site web du cabinet de recrutement Mozaïk RH. Consulté le 9 août 2009 à http://www.mozaikrh.com

National Center for Social Entrepreneurs (2001). Merging mission, market \& money - A non-profit's guide to social entrepreneurship. Consulté le 25 mai 2009 à http://www.ediblestrategies.com/fsd/2001_ENP_social_enterprise.pdf

Nicholls, A. (ed.) (2006) Social entrepreneurship: New models of sustainable social change, Oxford : Oxford University Press.

OCDE (Organisation de coopération et de développement économiques) (2001). Des citoyens partenaires, Paris : OCDE.

Painter, A. (2006). L'économie sociale au Canada--Concepts, données et mesure. Horizons, 8(2), 3034.

Paley, J. (2002).Towards an anthropology of democracy. Annual Review of Anthropology, (31), 469496.

Peredo, A.M. et McLean, M. (2006). "Social entrepreneurship": A critical review of the concept. Journal of World Business, 41(1), 56-65. 
Queinec, E. (2007). La croissance des ONG humanitaires. Une innovation devenue institution. Revue française de gestion, 8(177), 83-94.

Renaud, K. (2007), Capitalisme et entrepreneuriat social sont-ils compatibles? Le cas de la Grameen Bank. Bulletin CVRCE, No 97.

Robin des Bois (s.d.). Site web du restaurant Robin des Bois. Consulté le 27 novembre 2009 à http://www.robindesbois.ca

Salamon, L.M., Anheier, H.K., List, R., Toepler, S., Sokolowski, S,W., and Associates (1999). Defining the nonprofit sector: A cross-national analysis. Sterling : Kumarian Press.

Schlee, R.P., Curren, M.T. et Harich, K.R. (2009). Building marketing curriculum to support courses in social entrepreneurship and social venture competitions. Journal of Marketing Education, 31(1), 5-15.

Sullivan Mort, G., Weerawardena, J., et Carnegie, K. (2003). Social entrepreneurship: Towards conceptualization. International Journal of Nonprofit \& Voluntary Sector Marketing, 8(1), 76-88.

Tan, W.L., Williams, J., et Tan, T.M. (2005). Defining the "social" in "social entrepreneurship": Altruism and entrepreneurship. International Entrepreneurship and Management Journal, 1(3), 353-365.

Thompson, J., Alvy, G. et Lees, A. (2000). Social entrepreneurship--a new look at the people and the potential. Management Decision, 38(5), 328-338.

Ulhoi, JP (2005). The social dimensions of entrepreneurship. Technovation, 25(8), 939-946.

Valéau, P, Cimper, P. et Filion, L.J. (2004). Entrepreneuriat et organisations à but non lucratif (OBNL). Cahier de recherche 2004-10. Chaire d'entrepreneuriat Roger-J.A. Bombardier, HEC Montréal.

Waridel, L (2005). Acheter, c'est voter--Le cas du café. Montréal : Équiterre/Écosociété.

Warren ME (2008). Designing deliberative democracy. The British Columbia citizen's assembly. Coll. Theories of Institutional Design. Cambridge : Cambridge University Press.

Yunus, M (2006). Nobel lecture. Consulté le 26 mars 2009 à

http://nobelprize.org/nobel_prizes/peace/laureates/2006/yunus-lecture-en.html

Zhara, SA, Gedajlovic, E, Neubaum, DO et Shulman, JM (2006). Social Entrepreneurship : Domain, Contributions and Ethical Dilemmas. Actes de la Conference on Ethics and Entrepreneurship. University of Minnesota.

\section{Les auteurs}

François Brouard, DBA, CA, Sprott School of Business, Carleton University, Ottawa, Canada et Directeur, Centre Sprott pour les entreprises sociales (CSES). Email : francois_brouard@carleton.ca

Sophie Larivet, Dr Sc. gestion, Professeur-chercheure, École Supérieure du Commerce Extérieur, Paris, France et Membre, CEREGE et CSES. Email : sophie.larivet@esce.fr

Ouafa Sakka, Ph.D., Professeure adjointe. Sprott School of Business, Carleton University, Ottawa, Canada et Membre, CSES. Email : ouafa_sakka@carleton.ca 